\title{
Magnetic susceptibility of a neutron star crust
}

\author{
R D Blandford and L Hernquist \\ California Institute of Technology, Pasadena, California 91125, USA
}

Received 6 April 1982

\begin{abstract}
The magnetic susceptibility of the degenerate free electrons in the crust of a neutron star is computed for a range of densities, temperatures, and field strengths. It is shown that when the temperature is low enough (typically less than $10^{7} \mathrm{~K}$ for densities of about $10^{7} \mathrm{~g} \mathrm{~cm}^{-3}$ and $10^{12} \mathrm{G}$ fields), the susceptibility undergoes large de Haas-van Alphen oscillations. The crust is then unstable to the formation of layers of domains of alternating magnetisation. Associated with these domains are magnetic field fluctuations of a few per cent amplitude and anisotropic magnetostrictive stresses which may be large enough to crumble the crust. It is argued that these domains are unlikely to directly influence the surface properties of the neutron star but may possibly be coupled indirectly to observable effects.
\end{abstract}

\section{Introduction}

Observations of radio pulsars and pulsating $\mathrm{x}$-ray sources indicate that many neutron stars have surface flux densities $B \sim 10^{12} \mathrm{G}$. The outer crusts of neutron stars are believed to be in the form of a BCC ionic lattice supported against gravitational collapse by the pressure of effectively free degenerate electrons (e.g. Baym and Pethick 1975). At densities $\rho \geqslant 10^{5} \mathrm{~g} \mathrm{~cm}^{-3}$, electron-electron interactions and lattice corrections will have a negligible effect on the electron distribution function. The electron Fermi temperature $T_{\mathrm{F}}$ increases with depth $z$ below the surface and becomes relativistic at a density $\rho \sim 10^{6} \mathrm{~g} \mathrm{~cm}^{-3}$ and $z \sim 10 \mathrm{~m}$ for a standard $1.4 M_{\odot}, 10 \mathrm{~km}$ neutron star. The equilibrium ions range from ${ }^{56} \mathrm{Fe}$ to ${ }^{76} \mathrm{Fe}$ as the density increases from $10^{5} \mathrm{~g} \mathrm{~cm}^{-3}$ to $10^{11} \mathrm{~g} \mathrm{~cm}^{-3}$. We limit the discussion to densities $\rho \geqslant 10^{5} \mathrm{~g} \mathrm{~cm}^{-3}$ since the susceptibility at field strengths $\geqslant 10^{12} \mathrm{G}$ is effectively zero at lower densities. Possible observational consequences will be associated with effects at greater densities.

The magnetisation of the crust is generally very small compared with the magnetic field $H$. Nevertheless, when the electrons are sufficiently cool that their thermal energy is smaller than the spacing of the Landau levels, the magnetisation can undergo large de Haas-van Alphen oscillations with either changing field or changing Fermi energy. Under these conditions it sometimes becomes energetically favourable for the electron gas to separate into two phases containing different flux densities.

Earlier discussions of the magnetic susceptibility of neutron star crusts (e.g. Lee et al 1969, O'Connell and Roussel 1972, Schmid-Burgk 1973) have concentrated upon the discussion of whether or not the observed field may result from spontaneous magnetisation. This almost certainly cannot occur because the crust is insufficiently cool and, in any case, the magnetised state is at best metastable. In this paper it is assumed that the 
field is supported by conduction currents frozen into the interior of the star. The possibility that the field is produced by currents in the surface layers is currently under investigation (Blandford et al 1982).

In $\$ 2$ the calculation of free-electron magnetic susceptibility is reviewed and generalised. Domain structure and formation is discussed in $\S 3$ and the results are applied to neutron stars in $\S 4$. In $\S 5$ possible observational consequences are considered.

\section{Magnetic susceptibility of a free-electron gas}

\subsection{Magnetisation}

Consider a gas of free electrons in a quantising, homogeneous magnetic field of flux density $B$. Unless explicitly stated, we set $m_{\mathrm{e}}=c=k=1$ and measure $B$ in units of the critical field $B_{\mathrm{c}}=m_{\mathrm{e}}^{2} c^{3} e^{-1} \hbar^{-1}=4.41 \times 10^{13} \mathrm{G}$ and volume $V$ in units of $e^{2} \hbar^{2} m_{\mathrm{e}}^{-3} c^{-4}$. For the simple geometry under consideration the Dirac equation can be solved exactly (e.g. Berestetskii et al 1971) to give the energy spectrum:

$$
\varepsilon_{r}=\left(1+p_{\|}^{2}+2 r B\right)^{1 / 2} \quad r=0,1,2, \ldots
$$

The energy levels, which are known as Landau levels, are doubly degenerate for $r \neq 0$ and non-degenerate for $r=0$. The quantity $\varepsilon_{r}$, and hence the magnetisation, should be regarded as functions of $B$, and not $H$, because it is $B$ that is derived from the vector potential appearing in the Hamiltonian (e.g. Pippard 1980). This view has experimental support (e.g. Shoenberg 1962).

The magnetisation $M$ is conveniently calculated from the grand potential $\Omega$ which for small susceptibility is given by

$$
\Omega=-\alpha \frac{B V T}{4 \pi^{2}} \sum_{r=0}^{\infty} g_{r} \int_{-\infty}^{\infty} \mathrm{d} p_{\|} \ln \left\{1+\exp \left[\left(\mu-\varepsilon_{r}\right) / T\right]\right\}
$$

where $\alpha$ is the fine structure constant, $V$ is the volume, $g_{r}=2-\delta_{r 0}$ is the degeneracy, and $\mu$ is the chemical potential including rest mass (e.g. Lifshitz and Pitaevskii 1980). The Fermi temperature $T_{\mathrm{F}}$ is defined to be the temperature corresponding to the Fermi kinetic energy of the same density of electrons in the absence of a magnetic field, i.e.:

$$
T_{\mathrm{F}}=\mu-1+\frac{\pi^{2}}{6} \frac{T^{2}}{\mu} \frac{\left(2 \mu^{2}-1\right)}{\left(\mu^{2}-1\right)}+\mathrm{O}\left(T^{4}\right) .
$$

Now, the magnetisation $M$ is given by

$$
M=-\frac{1}{V}\left(\frac{\partial \Omega}{\partial B}\right)_{\mu, V, T}
$$

and the susceptibility $\chi=M / H \simeq M / B$ for the small values of concern to us. In addition, we will also be interested in the differential susceptibility, $\eta$, which is defined by:

$$
\eta=(\partial M / \partial B)_{T, V, \mu}
$$

Although, in general, $4 \pi|\chi| \ll 1$ for a relativistic electron gas, in certain regimes $4 \pi|\eta|$ can exceed unity, indicative of the possibility of the existence of magnetic domains.

The magnetisation is easily computed under three separate approximations which we now consider. 
2.1.1. Weak field $\left(B \ll \mu T \ll \mu T_{F}\right)$. When the thermal energy $(\sim T)$ is large compared with the spacing of the Landau levels $(\sim B / \mu)$ but small compared with the Fermi temperature, we can replace the sum in equation (2) by using the Euler-MacLaurin sum formula (e.g. Lifshitz and Pitaevskii 1980),

$$
\frac{1}{2} \sum_{r=0}^{\infty} g_{r} f(r)=\int_{0}^{\infty} f(s) \mathrm{d} s-\sum_{k=1}^{\infty} \frac{\mathscr{B} 2 k}{(2 k) !} f^{(2 k-1)}(0)
$$

where $\mathscr{B}_{2 k}$ are the Bernoulli numbers. The integral is independent of $B$ and does not contribute to the magnetisation. Ignoring a constant,

$$
\Omega=-\frac{\alpha V}{12 \pi^{2}} B^{2} \int_{0}^{\infty} \frac{\mathrm{d} p_{\|}}{\left(1+p_{\|}^{2}\right)^{1 / 2}\left[\exp \left\{\left(\varepsilon_{0}-\mu\right) / T\right\}+1\right]}+\mathrm{O}\left(B^{4}\right) .
$$

Using equation (4) to compute $M$ we obtain, after some manipulation,

$\chi=\frac{\alpha}{6 \pi^{2}} \ln \left[\mu+\left(\mu^{2}-1\right)^{1 / 2}\right]-\frac{\alpha \mu(\mu-1)^{1 / 2}}{36(\mu+1)^{3 / 2}}\left(\frac{T}{T_{\mathrm{f}}}\right)^{2}+\mathrm{O}\left(\frac{T}{T_{\mathrm{F}}}\right)^{4}+\mathrm{O}\left(B^{2}\right)$.

This is the relativistic generalisation of the standard non-relativistic susceptibility (e.g. Lifshitz and Pitaevskii 1980). The first term has been given previously by Rukhadze and Silin (1960). Note that non-relativistically only $\frac{1}{3}$ of the Pauli paramagnetic spin susceptibility is cancelled by the diamagnetic contribution from the orbital motion, whereas in the extreme relativistic limit, the purely paramagnetic part, $\chi_{\mathrm{p}}=\left(\alpha / 4 \pi^{2}\right) \mu\left(\mu^{2}-1\right)^{1 / 2}$, is almost completely cancelled. The magnetic susceptibility is always less than $10^{-3}$ in the weak-field limit.

2.1.2. Intermediate field $\left(\mu T<B \ll \mu T_{\mathrm{F}}\right)$. When the thermal energy is small compared with the Landau level spacing, $\Omega$ develops an oscillatory part $\hat{\Omega}$. As a result, the differential susceptibility can become large in magnitude. The computation of $\tilde{\Omega}$ is a straightforward relativistic generalisation of the calculation in Lifshitz and Pitaevskii (1980). We obtain:

$$
\tilde{\Omega}=\frac{\alpha B^{3 / 2} V T}{2 \pi^{2}} \sum_{k=1}^{\infty} \frac{\cos \left[2 \pi\left(k r_{\max }-\frac{1}{8}\right)\right]}{k^{3 / 2} \sinh \left(k T / T_{c}\right)}
$$

where $r_{\max }=\left(\mu^{2}-1\right) / 2 B$ is the Landau level of highest energy occupied and $T_{\mathrm{c}}=$ $B / 2 \pi^{2} \mu$ is the critical temperature for the appearance of oscillations. The oscillatory part of the susceptibility is given by:

$$
\tilde{\chi}=-\frac{\alpha T\left(\mu^{2}-1\right)}{2 \pi B^{3 / 2}} \sum_{k=1}^{\infty} \frac{\sin \left[2 \pi\left(k r_{\max }-\frac{1}{8}\right)\right]}{k^{1 / 2} \sinh \left(k T / T_{\mathrm{c}}\right)} .
$$

The non-oscillatory part is smaller than $\tilde{\chi}$ by $\sim O\left(r_{\max }^{-1 / 2}\right)$ and may be ignored. The quantity $\tilde{\chi}$ is still always less than $1 / 4 \pi$. For low temperature $T \ll T_{\mathrm{c}}, \tilde{\chi}$ becomes

$$
\bar{\chi}=-\frac{\alpha}{4 \pi^{3}} \frac{\left(\mu^{2}-1\right)}{\mu B^{1 / 2}} \sum_{k=1}^{\infty} \frac{\sin \left[2 \pi\left(k r_{\max }-\frac{1}{8}\right)\right]}{k^{3 / 2}} .
$$

the sum is bounded above by $\zeta\left(\frac{3}{2}\right)=2.61$. 
The oscillatory part of the differential susceptibility is:

$$
\tilde{\eta}=\frac{\alpha T\left(\mu^{2}-1\right)^{2}}{2 B^{5 / 2}} \sum_{k=1}^{\infty} \frac{k^{1 / 2} \cos \left[2 \pi\left(k r_{\max }-\frac{1}{8}\right)\right]}{\sinh \left(k T / T_{\mathrm{c}}\right)} .
$$

This quantity, which is approximately $r_{\max } \tilde{\chi}$, can exceed $1 / 4 \pi$.

2.1.3. Strong field $\left(\mu T \ll \mu T_{\mathrm{F}} \leqslant B\right)$. When $r_{\max }$, the number of Landau levels occupied, becomes small, the sum in equation (2) must be evaluated numerically. In the limit $B \rightarrow \infty$ all electrons occupy the ground level and the differential susceptibility vanishes.

In figure 1, we display the regions of validity of these three approximations.

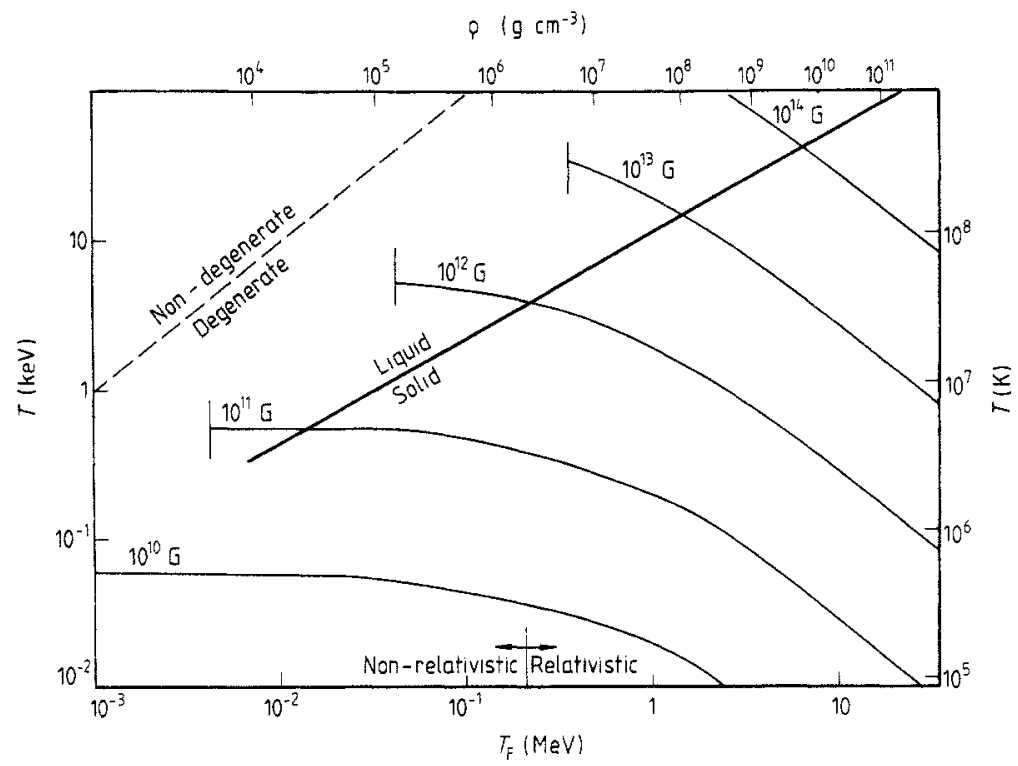

Figure 1. Weak-, intermediate-, and strong-field regions in the $T-T_{\mathrm{F}}$ plane. For each value of the flux density $B$ the curve separates the intermediate-field region where de Haas-van Alphen oscillations can occur from the weak-field region at higher temperature. Each line is terminated on the left by a vertical line denoting the boundary of the strong-field region. Also shown are the limit of the degenerate region (broken line) and the melting curve for neutron star crustal material (e.g. Flowers and Itoh 1981, Slattery et al 1980). The densities correspond to the lowest energy ionic lattice.

\subsection{Numerical evaluation}

We have computed the free-electron differential susceptibility for different combinations of the temperature and Fermi temperature. Four cases are plotted in figure 2. As the fiux density is increased into the intermediate field regime, de Haas-van Alphen oscillations set in and $|\tilde{\eta}|$ soon reaches its maximum value before declining $\propto B^{-3 / 2}$ at greater flux density. In the strong field region the period in $1 / B$ becomes comparable with $1 / B$. 

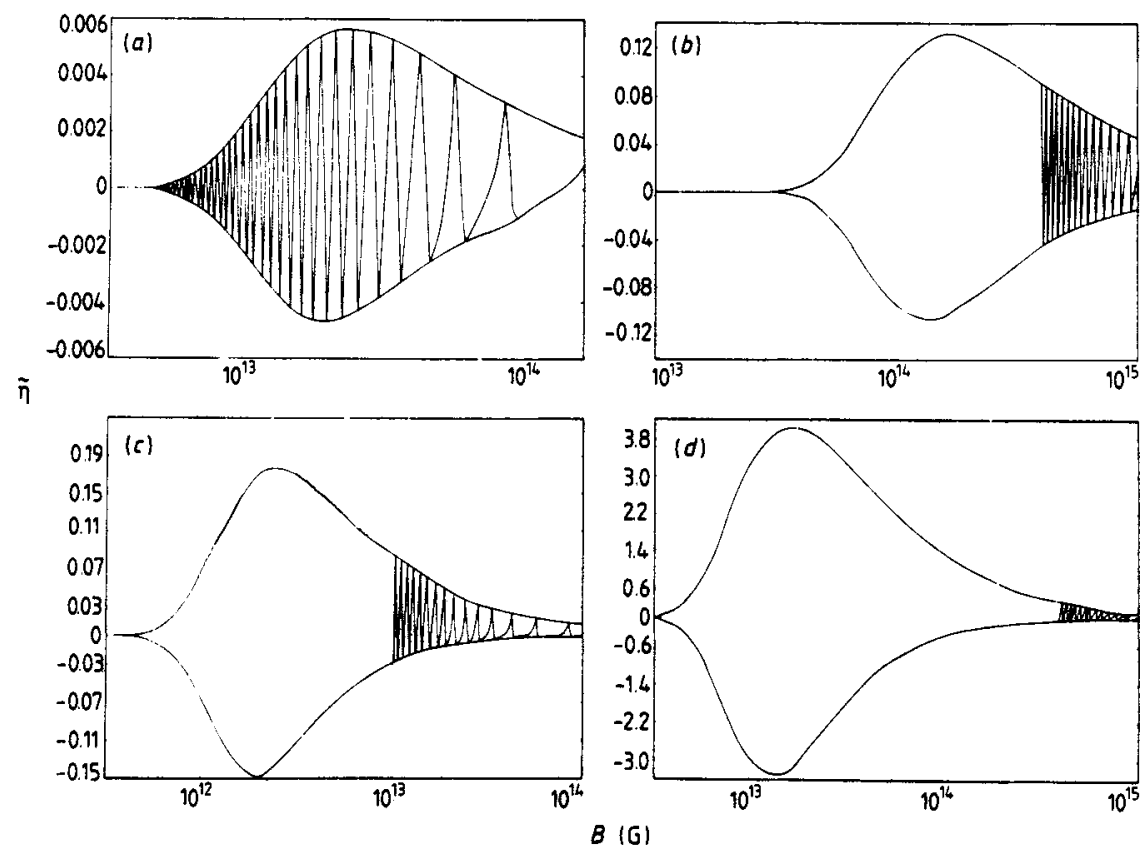

Figure 2. Differential susceptibility $\bar{\eta}$ as a function of $B$ for two values of both the Fermi temperature $T_{\mathrm{F}}$ and the temperature $T$. At low flux densities, where the de Haas-van Alphen oscillation frequency becomes large, only the envelope of the susceptibility is shown. (a) $T=10 \mathrm{keV}, T_{\mathrm{F}}=1 \mathrm{MeV} ;(b) T=10 \mathrm{keV}, T_{\mathrm{F}}=10 \mathrm{MeV} ;(c) T=1 \mathrm{keV}, T_{\mathrm{F}}=1 \mathrm{MeV} ;(d)$ $T=1 \mathrm{keV}, T_{\mathrm{F}}=10 \mathrm{MeV}$.

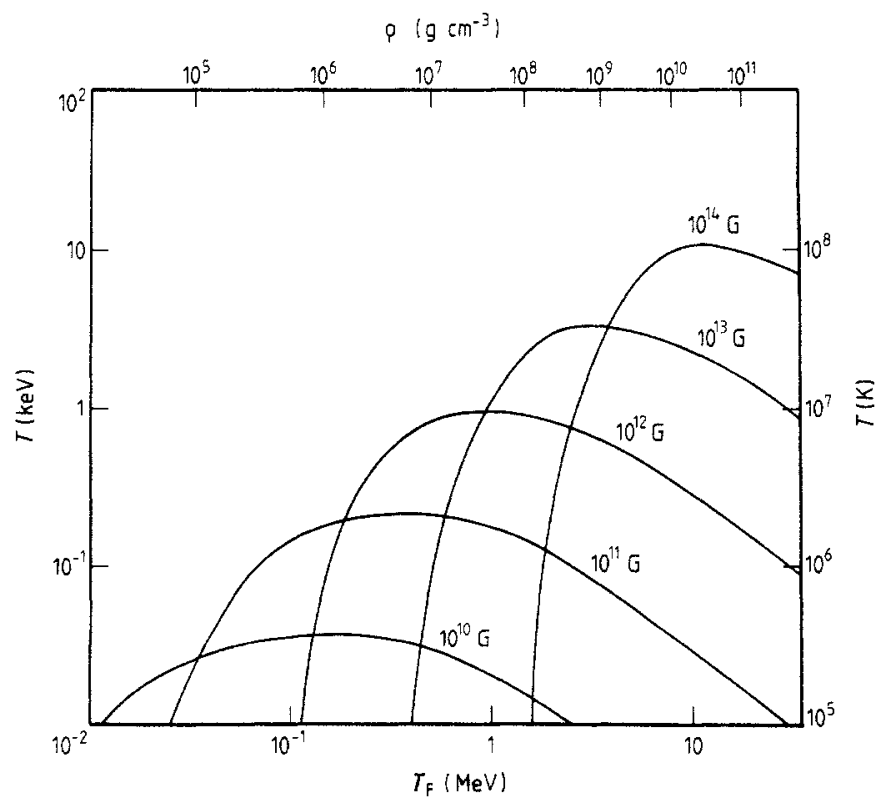

Figure 3. Region of $T-T_{\mathrm{F}}$ plane in which $4 . \pi \tilde{\eta}$ can exceed unity. When $T$ is less than the value indicated the electron gas is unstable to the formation of two phases of different magnetisation. 


\section{Domain formation}

For a bounded sample in a uniform field the magnetic field $H$ is related to the flux density $B$ by the relation

$$
H=B-4 \pi(1-n) M(B)
$$

where $n$ is the demagnetisation coefficient which is fixed by the shape of the sample. For our application to a thin neutron star crust, permeated by an approximately vertical field, $n \sim 0$ and will henceforth be ignored. When the differential susceptibility $\tilde{\eta}$ exceeds $1 / 4 \pi,(\partial H / \partial B)_{\mu, T}$ becomes negative, and the thermodynamic equilibrium becomes unstable (e.g. Lifshitz and Pitaevskii 1980). In fact, for the case $n=0$, as discussed at length by Pippard (1963,1980), Condon (1966), Azbel' (1970), the material will separate into two phases corresponding to different (usually anti-parallel) magnetisations.

In figure 3 we show the region of the $T-T_{\mathrm{F}}$ plane in which the electrons are unstable to phase separation for different values of the flux density. We see that for low enough temperature domain formation occurs over a density range limited at the low end by thermal effects and at the high end by the explicit dependence of $\tilde{\eta}$ upon the Fermi energy.

We may evaluate the change in magnetisation across a domain interface using the boundary conditions for $\boldsymbol{B}$ and $\boldsymbol{H}$. If the fractional change in $B$ is small and the field makes an angle $\theta$ with the interface, then

$$
\frac{H_{2}-H_{1}}{B_{2}-B_{1}} \approx-\tan ^{2} \theta
$$

where the subscripts 1 and 2 refer to the two domains. The field is deflected through an angle

$$
\delta \theta \simeq\left[\left(B_{2}-B_{1}\right) / B_{1}\right] \tan \theta
$$

at the interface. Equation (14) shows that the field $H$ must decrease as the flux density $B$ increases across the interface separating two domains.

A second relation between the flux density and the field strength is furnished by the 'equal area' rule:

$$
\int_{B_{1}}^{B_{2}} H(B) \mathrm{d} B=\frac{1}{2}\left(B_{2}-B_{1}\right)\left(H_{1}+H_{2}\right)
$$

where the integral is over the unstable portion of the $H(B)$ curve. Thus, given the angle $\theta$, both $B_{1}$ and $B_{2}$ are fixed by equations (14) and (16) and the shape of the magnetisation curve. Note that there is a maximum allowed angle $\theta$.

If the electron gas is to be regarded as a fluid in thermodynamic equilibrium, i.e. there are no strains in the ionic lattice communicated to the electron gas by electric fields, then there is a third condition which must be satisfied. The pressure, which is hydrostatic and is given by $-\Omega / V$, must be the same on either side of the domain interface. This implies that

$$
\int_{B_{1}}^{B_{2}} M \mathrm{~d} B=0
$$

or

$$
B_{1}+B_{2}=H_{1}+H_{2}
$$




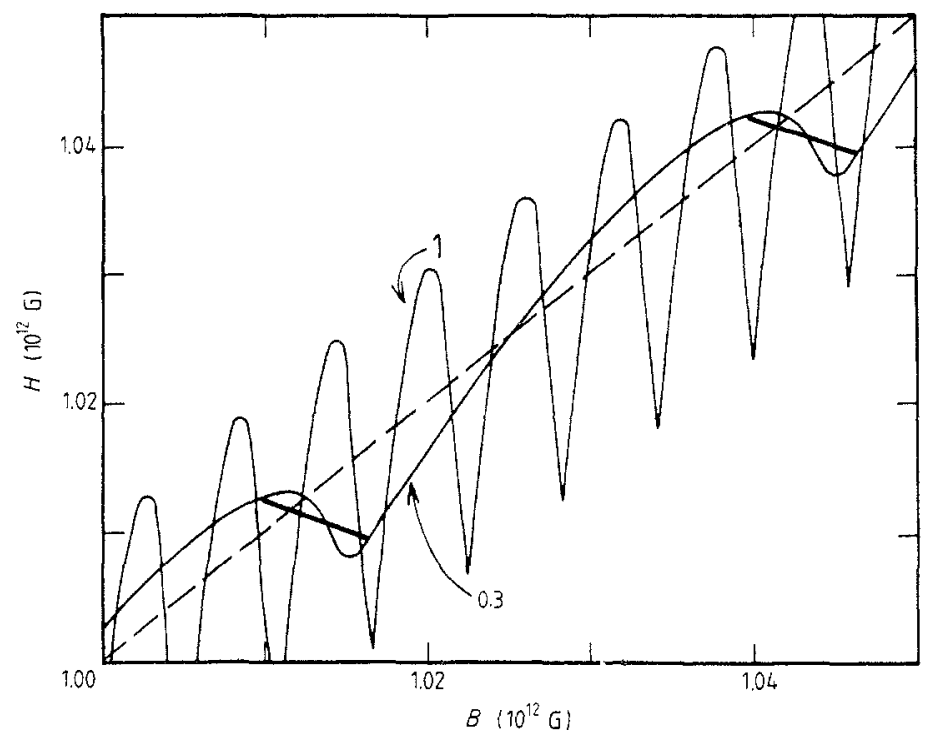

Figure 4. $H(B)$ curve for $T=0.1 \mathrm{keV}$ and flux density $B$ near $10^{12} \mathrm{G}$ for electron gases of Fermi temperatures 0.3 and $1 \mathrm{MeV}$. The corresponding densities are $\rho=3.9 \times 10^{6}$, $4.5 \times 10^{7} \mathrm{~g} \mathrm{~cm}^{-3}$, respectively. Also shown is the line (broken) representing $B=H$. Both curves are unstable and the phase diagram for $T=0.3 \mathrm{MeV}$ is drawn, assuming an angle of $30^{\circ}$ between the domain interface and the field.

using equations (4) and (16). In this case equation (18) should apply and the angle $\theta$ will be fixed at some non-zero value.

In figure 4 we show the $H(B)$ relation for an electron gas at a temperature $T=$ $0.1 \mathrm{keV}$ for two different Fermi temperatures.

\section{Neutron star crusts}

\subsection{Structure}

As can be seen from figure 3, domain formation should occur in the crust of magnetised $\left(B \sim 10^{12} \mathrm{G}\right)$ neutron stars at densities $\rho \sim 10^{6} \mathrm{~g} \mathrm{~cm}^{-3}$ below temperatures $T \sim 10^{7} \mathrm{~K}$. According to cooling calculations (e.g. Tsuruta 1981), the crust should become sufficiently cool after about $10^{4}$ years, a time much shorter than a typical radio pulsar age $\left(\sim 3 \times 10^{6}\right.$ years $)$.

The variation of the magnetic susceptibility is, however, crucially different from the usual situation encountered in solid state physics in which oscillatory magnetisation appears as $H$ is varied. In a neutron star crust, we expect that $H$ will be determined by currents which flow within a region about $10 \mathrm{~km}$ in size and that the field is locally constant. However, as the depth $z$ below the surface increases, the Fermi temperature $T_{\mathrm{F}}$ increases and it is this variation that is responsible for the oscillatory magnetisation.

The thickness of the crust is determined by the surface gravity $g=10^{14} \mathrm{~g}_{14} \mathrm{~cm} \mathrm{~s}^{-2}$. It can be shown that for degenerate electron pressure support,

$$
z=\frac{1}{m_{\mathrm{p}} g} \int \frac{\mathrm{d} T_{\mathrm{F}}}{\mu_{\mathrm{e}}} \simeq 50\left(\frac{T_{\mathrm{F}}}{1 \mathrm{MeV}}\right) g_{14}^{-1}\left(\frac{\mu_{\mathrm{e}}}{2}\right)^{-1} \mathrm{~m}
$$


where $\mu_{\mathrm{e}}$ is the mean molecular weight per electron which varies between about 2 and 3 in the crust (e.g. Baym and Pethick 1975).

At the surface density $\rho \sim 10^{4} \mathrm{~g} \mathrm{~cm}^{-3}$ for a field $B=10^{12} B_{12} \mathrm{G}$ all the electrons will be in the ground-state Landau orbital. However, as the density increases, the number of Landau orbitals $r_{\max } \approx 20\left(\mu^{2}-1\right) B_{12}^{-1}$ will also increase. The spacing between layers associated with successive integral values of $r_{\max }$ is

$$
\Delta z=\frac{B}{\mu} \frac{\mathrm{d} z}{\mathrm{~d} \mu}=\frac{B}{\mu \mu_{\mathrm{e}} m_{\mathrm{p}} g} \approx 55 \mathrm{~g}_{14}^{-1}\left(\frac{\mu_{\mathrm{e}}}{2}\right)^{-1} \mu^{-1} B_{12} \mathrm{~cm} .
$$

At non-relativistic Fermi temperatures, the spacing is constant. If the temperature is low enough for domains to form, then $\Delta z$ is the average vertical spacing between domain interfaces. In this case, the horizontal variation in the field will be

$$
\frac{\delta B}{B}=\frac{2 B \beta}{\left(\mu^{2}-1\right)} \simeq 0.48 B_{12}\left(\mu^{2}-1\right)^{-1} \beta
$$

where $\beta \leqslant 1$ is the strength of the oscillation relative to the maximum allowed oscillation. For $T \ll T_{c}, \beta \sim 1$.

The actual size and shape of the domains is problematical. There seem to be two possibilities. The first is that the domains have a horizontal scale $\sim \Delta z$ which is in general required if there is to be a balance in the electron pressures. The second possibility is that the domains form a two-dimensional lattice of vertical needles of thickness given roughly by the geometrical mean of the Larmor radius and $\Delta z \sim 10^{-4} \mathrm{~cm}$. This second configuration minimises the sum of magnetic and surface energy (e.g. Pippard 1980). The choice between these two possibilities is probably governed by the magnetisation history and the ability of the lattice to withstand stresses (see $\$ 4.4$ below).

In either case, unless there is some magnetisation structure on a length scale comparable with the depth $z$, there should not be any influence on the surface field as the amplitude of the field perturbation will be smaller than that given by equation (21) by a factor $\sim \exp (-\pi z / \Delta z)$.

\subsection{Collisional broadening}

Electron scattering will broaden the Landau levels and may damp the de Haas-van Alphen oscillations (Dingle and Shoenberg 1950, Springford 1980). Collisional broadening is negligible when the collision frequency $\nu_{\mathrm{c}}$ is much smaller than the gyro frequency $e B / \mu$. An approximate non-relativistic calculation gives the result that the terms in the sum in equation (9) must each be multiplied by a factor exp $\left[-k \pi \nu_{\mathrm{d}} / e B\right]$, (Higgins and Lowndes 1980). The collision frequency has been computed by Urpin and Yakovlev (1980) and Yakovlev and Urpin (1980a, b). For solid iron, neglecting impurity scattering, $\nu_{\mathrm{c}} \sim 3 \times 10^{16}\left(T / 10^{6} \mathrm{~K}\right) \rho_{6}^{-1 / 3} \mathrm{~Hz}$. By inspection of figure 1 , we see that electron collisions should not affect the de Haas-van Alphen oscillations.

\subsection{Eddy currents}

As the neutron star crust cools and regions of previously uniform magnetisation become unstable, internal conduction currents will grow and lead to the creation of domains on the ohmic dissipation time, $t_{\mathrm{d}}$. Using Urpin and Yakovlev's (1980) conductivity for a solid crust, we obtain

$$
t_{\mathrm{d}} \sim 3 \times 10^{5}(w / 1 \mathrm{~m})^{2} \rho_{6}^{4 / 3} T_{6}^{-1} \mathrm{~s}
$$


where $w$ is the horizontal length scale for field variation. We have assumed that the geometry is such as to allow the creation of a Hall field. Again, we have ignored possible impurities and lattice defects. We can also use equation (22) to verify that small scale conduction currents in the outer crust $\left(\rho \leqslant 10^{8} \mathrm{~g} \mathrm{~cm}^{-3}\right)$ will probably have decayed. (See Blandford et al (1982) for an alternative viewpoint.)

\subsection{Magnetostriction}

As can be seen from figures 1 and 3 the crust will be solid whenever domain formation is possible. In general the formation or adjustment of domain structure involves anisotropic magnetostrictive stresses $\sim 2 \pi M^{2}$ (see Appendix). These will be balanced by elastic stresses in the lattice provided that they do not exceed the yield stress for shear flow $Y$. Using Ruderman (1972), we estimate

$$
Y \sim Z^{2} e^{2}\left(\rho / A m_{\mathrm{p}}\right)^{4 / 3} \varepsilon \sim 4 \times 10^{18} \rho_{6}^{4 / 3} \varepsilon_{-3} \text { dyn } \mathrm{cm}^{-2}
$$

where $\varepsilon=10^{-3} \varepsilon_{-3}$ is the maximum allowed strain angle. Unless the crystal is unusually pure, we expect that $\varepsilon_{-3} \sim 1$. Using equation (11) magnetic stresses are roughly $2 \pi \tilde{\chi}^{2} B^{2} \approx \alpha^{2} 2^{-4} \pi^{-6}(2.61)^{2}\left(u^{2}-1\right)^{2} \mu^{-2} B$, implying that the maximum flux density which the crustal lattice can withstand satisfies:

$$
B_{\max 12} \sim\left(1+0.6 \rho_{6}^{2 / 3}\right) \varepsilon_{-3} .
$$

If $B>B_{\max }$, the crust will crumble and the domains will adjust to a lower energy state on the eddy current decay time given by equation (22). The domain structure will be constrained by electron pressure balance.

\section{Discussion}

Most old radio pulsars appear to have surface flux densities in the range $1-5 \times 10^{12} \mathrm{G}$ (e.g. Manchester 1981) and surface gravities $\sim 2 \times 10^{14} \mathrm{~cm} \mathrm{~s}^{-2}$ (e.g. Bahcall 1978). We expect the outer crust to be isothermal with a temperature $T \leqslant 10^{6} \mathrm{~K}$ (e.g. Arons 1981). Therefore domains will form below a depth $z \sim 5-10 \mathrm{~m}$ with a vertical spacing $\Delta z \sim 20-50 \mathrm{~cm}$.

Old pulsars often show complex and stable pulse profiles. There is no well-accepted theory of pulsar radio emission, but it is widely believed that the pulse structure has its origin in quasi-permanent magnetic or topographic features or the neutron star surface. These features indicate the places where current is preferentially drawn from the stellar surface (e.g. Ruderman 1981). It is intriguing to enquire if they could be connected in any way with sub-surface domain structure.

This connection is unlikely to be direct unless the domains have horizontal structure on a length scale $\sim z$, since structure on smaller scales will lead to exponentially small surface effects. Further, the maximum free energy associated with the domains $\left(\sim 10^{20}\right.$ erg per domain $)$ is very small by neutron star standards.

However, there are some possibilities for an indirect connection. The current believed to be flowing through the polar cap of a pulsar with period $\sim 0.3-3 \mathrm{~s}$ is roughly $I \sim 10^{11}-10^{12} \mathrm{~A}$. If this current crosses the field at a depth $d$ then the associated magnetic perturbation is $\delta B \sim 10^{10}\left(I / 10^{12} \mathrm{~A}\right)(\mathrm{d} / 1 \mathrm{~m})^{-1} \mathrm{G}$. The depth to which the currents flow is determined by the surface potential variation and the time that has elapsed since the potential was established. Presumably the currents flow over a region as large as the 
polar cap about $100 \mathrm{~m}$ in size. However, for the currents to penetrate to a depth of about $100 \mathrm{~m}$ requires $\sim 10^{6} T_{6}^{-1}$ years, using equation (22). If these currents flow at depths in the range $10-100 \mathrm{~m}$ then they may be responsible for establishing large-scale patterns of magnetisation.

Secondly, the fact that the maximum field given by equation (24) is close to the observed surface field suggests that the crustal regions are seismically active. Any sudden readjustment of the domain structure will cause a local departure from isostacy which will be relieved on the ohmic dissipation timescale. At the surface this time is of the order of a few seconds, which is a characteristic timescale for marching subpulses.

Finally, there will be small-scale fluctuations in the transport properties of the crust because of the domains. If the domains are arranged regularly, then it is possible that they cause thermal or potential patterns on the surface.

However, we have no more specific proposals for the possible observational consequences of domain formation. It is most likely that their effects are negligible.

\section{Acknowledgments}

We thank Nordita and the Institute of Astronomy for hospitality and financial support. We acknowledge helpful discussions with E Gudmundsson, C Pethick and S Tsuruta. The support of the National Science Foundation [AST80-17752] and the Alfred P Sloan Foundation is gratefully acknowledged.

\section{Appendix}

Some confusion has arisen in the literature concerning the pressure of a degenerate electron gas in a magnetic field (e.g. Canuto and Chiu 1971, Schmid-Burgk 1973). From a thermodynamic point of view, the pressure can be derived from the grand potential as

$$
P=-\left(\frac{\partial \Omega}{\partial V}\right)_{u, T, B}=-\frac{\Omega}{V} .
$$

This pressure is isotropic. However, the kinetic pressure of the electrons is anisotropic. The components resolved parallel and perpendicular to the field direction can be expressed as follows:

$$
\begin{aligned}
P_{\|}^{k} & =\int_{-\infty}^{\infty} \mathrm{d} p \sum_{r=0}^{\infty} \frac{\alpha \beta}{4 \pi^{2}} g_{r} \frac{P_{\|}^{2}}{\varepsilon_{r}}\left[\exp \left(\varepsilon_{r}-\mu\right) / T+1\right]^{-1} \\
& =-\Omega / V \\
P_{\perp}^{k} & =\int_{-\infty}^{\infty} \mathrm{d} p_{\|} \sum_{r=1}^{\infty} \frac{\alpha \beta}{4 \pi^{2}} g_{r} \frac{\left\langle p_{\perp}^{2}\right\rangle}{\varepsilon_{r}}\left[\exp \left(\varepsilon_{r}-\mu\right) / T+1\right]^{-1} .
\end{aligned}
$$

Now $\left\langle p_{\perp}^{2}\right\rangle=r B$, averaging over the two spin states. Equation (A3) can be related to equation (2) after an integration by parts by

$$
P_{\perp}^{k}=\frac{B^{2}}{V}\left(\frac{\partial}{\partial B}(\Omega / B)\right)_{\mu, V, T}=-\frac{\Omega}{V}-M B .
$$


However if we compress the electron gas perpendicular to $B$ then we must also do work against the Lorentz force density involving the magnetisation current density, $(\nabla \times M) \times B$. There is therefore an additional magnetic contribution to the perpendicular pressure of magnitude $M B$ which cancels the second term in (A4). The composite pressure tensor is therefore isotropic, in agreement with the thermodynamic result, equation (A1).

In a body of arbitrary shape, there will be additional anisotropic stresses $\sim 2 \pi M^{2}$ associated with the demagnetisation field. In a solid these will be balanced by elastic stresses.

\section{References}

Arons J 1981 Astorophys J. 2481009

Azbel' M Ya 1970 Sov. Phys.-Usp. 12507

Bahcall J N 1978 Ann. Rev. Astron. Astrophys. 16241

Baym G and Pethick C 1975 Ann. Rev. Nucl. Sci. 2527

Berestetskii V B, Lifshitz E M and Pitaevskii L P 1971 Relativistic Quantum Theory (Oxford: Pergamon)

Blandford R B, Applegate J and Hernquist L 1982 Mon. Not. R. Astron. Soc (submitted)

Canuto V and Chiu H Y 1971 Space Sci. Rev. 123

Condon J H 1966 Phys. Rev. 145526

Dingle R B and Shoenberg D 1950 Nature 166652

Flowers $E$ and Itoh 1981 preprint

Higgins R J and Lowndes D H 1980 in Electrons at the Fermi Surface, ed. M Springford (Cambridge: Cambridge University Press)

Lee H J, Canuto V, Chiu H Y and Chiuderi C 1969 Phys. Rev. Lett. 23390

Lifshitz E M and Pitaevskii L P 1980 Statistical Physics (Oxford: Pergamon)

Manchester R N 1981 in Pulsars ed. W Sieber and R Wielebinski (Dordrecht: Reidel)

O'Connell R F and Roussel K M 1972 Astron. Astrophys. 18198

Pippard A B 1963 Proc. R. Soc. A 272192

1980 in Electrons at the Fermi Surface ed M Springford, (Cambridge: Cambridge University Press)

Ruderman M A 1972 Ann. Rev. Astron. Astrophys. 10427

1981 in Pulsars, ed. W Sieber and R Wielebinski (Dordrecht: Reidel)

Rukhadze A A and Silin V P 1960 Sov. Phys.-JETP 11463

Schmid-Burgk J 1973 Astron. Astrophys. 26335

Schoenberg D 1962 Phil. Trans, R. Soc. A 25585

Slattery W L, Doolen G D and De Witt H E 1980 Phys. Rev. A 212087

Springford M 1980 in Electrons at the Fermi Surface ed M Springford (Cambridge: Cambridge University Press)

Tsuruta S 1981 in Pulsars ed. W Sieber and R Wielebinski (Dordrecht: Reidel)

Urpin V A and Yakovlev D G 1980 Sov. Astron. 24425

Yakovlev D G and Urpin V A 1980a Sov. Astron. 24126

- 1980b Sov. Astron. 24303 